\title{
Uplink Detection and BER Analysis for Ambient Backscatter Communication Systems
}

\author{
Gongpu Wang ${ }^{\dagger}$, Feifei Gao ${ }^{\ddagger}$, Zhongzhao Dou*, and Chintha Tellambura* \\ ${ }^{\dagger}$ School of Computer and Information Technology, Beijing Jiaotong University, Beijing, China \\ ${ }^{\ddagger}$ Department of Electrical Engineering, Tsinghua University, Beijing, China \\ ${ }^{\star}$ School of Electronic and Information Engineering, South China University of Technology, Guangzhou, China \\ *Department of Electrical and Computer Engineering, University of Alberta, Edmonton, Canada \\ Email: gpwang@bjtu.edu.cn, feifeigao@ieee.org, sinceredzz@gmail.com, chintha@ece.ualberta.ca
}

\begin{abstract}
Ambient backscatter is a new communication technology that utilizes ambient radio frequency signals to enable battery-free devices to communicate with each other. In this paper, we study the problem of signal detection and bit error rate (BER) performance for this new communication system where the differential encoding is adopted to eliminate the necessity of channel estimation. We formulate a new transmission model, design the data detection approach, and derive the optimal/approximate closed-form detection thresholds. In addition, the performance at high signal-to-noise region (SNR) is also analyzed, where the lower and the upper bounds of BERs are found. Simulation results are then provided to corroborate our theoretical studies.
\end{abstract}

\section{INTRODUCTION}

Radio Frequency Identification (RFID) systems have attracted increasing attentions from both academic circles and industrial communities over the past two decades [1]. A typical passive RFID system mainly consists of a reader (or named as an interrogator) and a tag (also known as transponder). The reader first generates an electromagnetic wave, and the tag receives and backscatters the wave with modulated information bits to the reader.

Clearly, one essential physical-layer technology for passive RFID systems is radio backscatter, a type of wireless communication by means of reflection rather than radiation. The origin of radio backscatter dates back to World War II when the backscattered radio signal was used to identify one coming airplane as "friend or foe" [2]. The first literature work about backscatter communication was given by Harry Stockman in 1948 [3]. From then on, backscatter has been continuously studied and the corresponding RFID products have been developed mainly in identification/supply chain applications. From 1990 to 2000, one famous and successful application of RFID systems is Electronic Toll Collection (ETC). After 1990s, the rapid progress in integrated circuits resulted in dropping tag costs, which enabled the wide-spread usage of RFID products and also aroused significant interests on further investigation of the backscatter technology [4]. Recently, the backscatter technology has been exploited for Internet of things (IoT) due to its capability in reducing energy and monetary cost of sensors [1], [6].

As shown in Fig. 1(a), the traditional backscatter requires that the reader generate a carrier wave which will be received
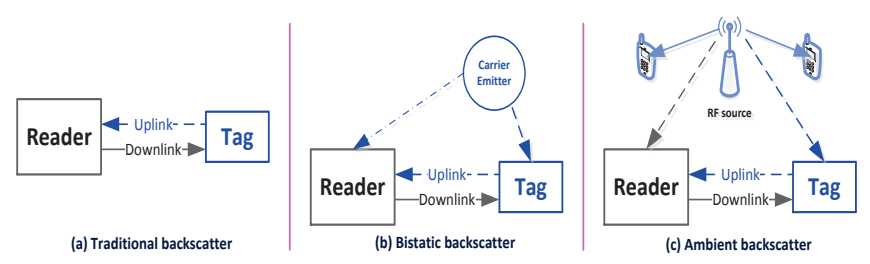

Fig. 1. Three types of backscatter.

and remodulated by the tag [4]. Therefore, the backscattered wave will suffer from a round-trip path loss, which will impose an limit on the communication distance [4], [6].

To further increase the field coverage and communication range, another two types of scatter are newly proposed: bistatic scatter [6] and ambient backscatter [7], [8]. Bistatic scatter is suggested in the reference [6] through dislocating the carrier emitter from the reader (Fig. 1(b)). Differently, ambient backscatter utilizes ambient radio frequency (RF) signals, such as television (TV) radio, to enable battery-free tag to communicate with the reader [7], [8]. As depicted in Fig. 1(c), the tag was motivated by certain ambient wireless signals, instead of fixed-frequency sine/cosine waves.

The key idea of the ambient backscatter can be described as follows: (i) the battery-free devices can transmit 0 or 1 bit through switching the antenna between reflecting and nonreflecting states, i.e., a change of the tag antenna impedance states; (ii) the transmitter can backscatter information at a much lower data rate than the ambient signals so that the receiver can separate the two signals. Based on such idea, the authors in [7] devised a prototype that two battery-free devices can communicates via ambient backscatter. In 2014, a communication system, named as Wi-Fi backscatter, was designed to connect the battery-free devices with off-the-shelf Wi-Fi devices [8].

Ambient backscatter, as a new communication technology, can enable ubiquitous communication among pervasive devices, liberate sensor nodes from maintenance-heavy batteries [7], and even may bring a new generation of RFID products [9]. Nevertheless, the whole theory such as signal processing and performance analysis for ambient backscatter communication systems is different from that for the existing communication systems and there exist many open problems 


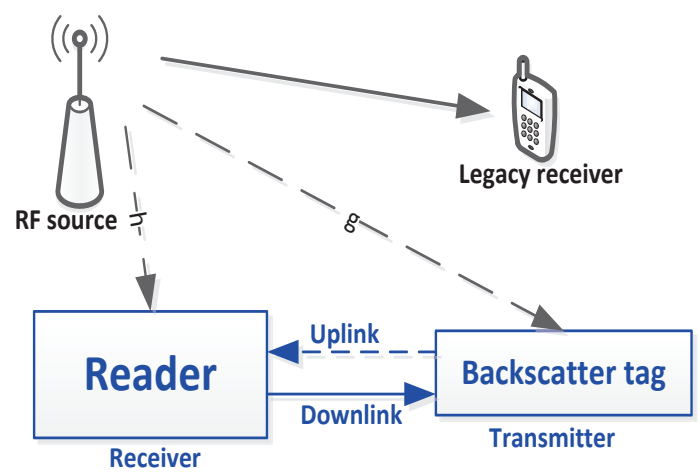

Fig. 2. A communication system of RF-powered devices that utilizes ambient backscatter.

that worth further investigation.

In this work, we study signal detection and examine the corresponding BER performance for ambient backscatter communication systems. We formulate a theoretical system model and design the data detection approach without channel state information (CSI). The corresponding BER performance is also analyzed and a simplified BER expression, as well as its upper and lower bounds are derived in high signal-to-noise ratio (SNR) region. Finally, simulation results are provided to verify the proposed studies.

\section{SYSTEM MODEL}

Consider an ambient backscatter communication system consisting of one reader and one tag, as shown in Fig. 2. Different from conventional RFID tag, the tag here utilizes the RF signals from other wireless communication systems to communicate with the reader. Suppose the signal transmitted by the RF source is $s(n) e^{j 2 \pi f_{s} n}$ where $f_{s}$ denotes the carrier frequency of the RF source and $s(n)$ is the complex baseband equivalent signal. The tag will receive the RF signal from the $\mathrm{RF}$ source and will transmit its own binary signal $B(n)$ to the reader through backscattering the signal $s(n)$ or not. If $B(n)=0$, the tag changes its impedance so that little energy of $x(n)$ can be reflected, while if $B(n)=1$, the tag switches the impedance so that the signal can be scattered and the reader will receive the scattered signal [7].

To avoid sending training sequence that is power-consuming and complexity-increasing for the battery-free tag, differential encoding is adopted at tag. Specifically, an information bit 0 corresponds to the same state in two consecutive intervals, while an information bit 1 corresponds to the transfer from non-backscatter to backscatter or from backscatter to nonbackscatter. This differential encoding is realized by a differential encoder before the modulator as shown in Fig. 3. Mathematically, the relationship between the input $A(k)$ and the output $B(k)$ of the differential encoder can be expressed as

$$
B(k)=A(k) \otimes B(k-1),
$$

where $\otimes$ denotes addition modulo 2 .

Denote the channel between the RF source and the reader as $h$, the channel between the RF source and the reader as $g$, and the channel between the reader and the tag as $\zeta$. The

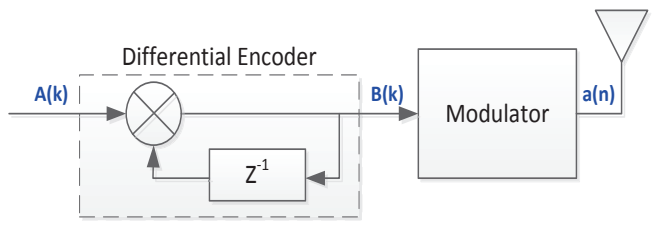

Fig. 3. Differential encoder of the backscatter tag.

channels $h, g$, and $\zeta$ are assumed as slow-fading and remain unchanged during at least two consecutive intervals of $B(k)$.

The signal received by the tag can be expressed as as [6]

$$
x(n)=g s(n) e^{j 2 \pi f_{s} n} .
$$

Noting that there may exist some carrier phase offset between the signal $x(n)$ and $s(n)$, and it can be absorbed into the complex channel $g$.

Meanwhile, the signal backscattered by the tag can be written as

$$
a(n)=\eta B(n) x(n), \quad B(n)=0,1
$$

where $\eta$ denotes the complex attenuation of the signal $x(n)$ inside the tag. Note that the data rate of the signal $B(n)$ is much less than that of the RF signal $x(n)$, and $B(n)$ will remain unchanged during $N$ symbols of $x(n)$. That is, $B(k N+j)$ will be the same for $j=1,2, \cdots, N$.

Therefore, the reader will receive the passband signal

$$
\widetilde{y}(n)=h s(n) e^{j 2 \pi f_{s} n}+\zeta a(n)+\widetilde{w}_{b}(n)
$$

where $\widetilde{w}_{b}(n)$ is the zero-mean additive white Gaussian noise (AWGN) at the reader with variance $N_{w b}$.

Define the carrier frequency generated at the reader as $f_{r}$ and the carrier phase offset between the RF source and the reader as $\theta_{0}$. After demodulating the received passband signal $\widetilde{y}(n)$ with the carrier signal $e^{-j\left(2 \pi f_{r} n+\theta_{0}\right)}$, the reader will obtain the baseband signal

$$
\begin{aligned}
y(n)= & \widetilde{y}(n) e^{-j\left(2 \pi f_{r} n+\theta_{0}\right)} \\
= & h s(n) e^{-j\left(2 \pi \Delta_{f} n+\theta_{0}\right)} \\
& +\eta \zeta g B(n) s(n) e^{-j\left(2 \pi \Delta_{f} n+\theta_{0}\right)}+w_{b}(n),
\end{aligned}
$$

where $\Delta_{f}=f_{s}-f_{r}$ denotes the carrier frequency offset (CFO) and $w_{b}(n)=\widetilde{w}_{b}(n) e^{-j\left(2 \pi f_{r} n+\theta_{0}\right)}$ is an equivalent noise with the same variance $N_{w b}$.

The main task of reader is to recover $B(n)$ from the baseband signal $y(n)$ without any channel knowledge and CFO knowledge.

Remark 1: Following the analysis in the reference [5], the signal received in the reader is modeled as

$$
\widetilde{y}(n)=h s(n) e^{j 2 \pi f_{s} n}+\zeta a(n)+\zeta \widetilde{w}_{a}(n)+\widetilde{w}_{b}(n),
$$

where $w_{a}(n)$ denotes the AWGN at the tag and is scattered back to the reader. However, the tag circuit consists only of passive components and takes little signal processing operations. In fact, the replica of the transmitted signal from the reader is provided to the tag through radiative coupling, and hence the thermal noise can be negligible, i.e., $w_{a}(n) \approx 0$. A detailed analysis about the backscattered radiated field and the channel modelling can be found in [4], [6], [10]. 


\section{Signal Detection at the Reader}

Let us rewrite (6) as

$$
y(n)= \begin{cases}h s(n) e^{-j\left(2 \pi \Delta_{f} n+\theta_{0}\right)}+w_{b}(n), & B(n)=0 \\ \mu s(n) e^{-j\left(2 \pi \Delta_{f} n+\theta_{0}\right)}+w_{b}(n), & B(n)=1\end{cases}
$$

where $\mu \triangleq h+\eta \zeta g$ denotes the combined channel information.

Suppose the reader totally receives $K N$ samples. It then compute the average power of the $N$ samples of $y(n)$ that corresponding to each single backscattered bit as

$$
\Gamma_{k}=\frac{1}{N} \sum_{n=(k-1) N+1}^{k N}|y(n)|^{2}, \quad 1 \leq k \leq K .
$$

It can be easily found that

$$
\Gamma_{k}= \begin{cases}\Gamma_{k, 0}=M_{k, 0}+L_{k, 0}, & B(n)=0 \\ \Gamma_{k, 1}=M_{k, 1}+L_{k, 1}, & B(n)=1\end{cases}
$$

where

$$
\begin{aligned}
M_{k, 0} & =\sum_{n=(k-1) N+1}^{k N} \frac{|h|^{2}|s(n)|^{2}+\left|w_{b}(n)\right|^{2}}{N}, \\
M_{k, 1} & =\sum_{n=(k-1) N+1}^{k N} \frac{|\mu|^{2}|s(n)|^{2}+\left|w_{b}(n)\right|^{2}}{N}, \\
L_{k, 0} & =\frac{1}{N} \sum_{n=(k-1) N+1}^{k N} 2 \Re\left\{h s(n) e^{-j\left(2 \pi \Delta_{f} n+\theta_{0}\right)} w_{b}^{H}(n)\right\}, \\
L_{k, 1} & =\frac{1}{N} \sum_{n=(k-1) N+1}^{k N} 2 \Re\left\{\mu s(n) e^{-j\left(2 \pi \Delta_{f} n+\theta_{0}\right)} w_{b}^{H}(n)\right\} .
\end{aligned}
$$

When $N$ is a large number, the following approximation can be made:

$$
\begin{aligned}
& M_{k, 0} \approx|h|^{2} P_{s}+N_{w b}, \\
& M_{k, 1} \approx|\mu|^{2} P_{s}+N_{w b},
\end{aligned}
$$

where $P_{s}$ is the average power of the RF source signal $s(n)$. Assuming that the slow-fading channels $h, \zeta$ and $g$ remain unchanged during some consecutive intervals of transmitting $B(k-1)$ and $B(k)$, we can obtain from the central limit theorem (CLT) that $L_{k, 0} \sim \mathcal{N}\left(0, \varsigma_{0}^{2}\right)$ and $L_{k, 1} \sim \mathcal{N}\left(0, \varsigma_{1}^{2}\right)$, where the variances are given by

$$
\varsigma_{0}^{2}=\frac{2}{N}|h|^{2} P_{s} N_{w b}, \quad \varsigma_{1}^{2}=\frac{2}{N}|\mu|^{2} P_{s} N_{w b} .
$$

Therefore, we can obtain

$$
\Gamma_{k}=\left\{\begin{array}{l}
\Gamma_{k, 0} \sim \mathcal{N}\left(|h|^{2} P_{s}+N_{w b}, \varsigma_{0}^{2}\right) \\
\Gamma_{k, 1} \sim \mathcal{N}\left(|\mu|^{2} P_{s}+N_{w b}, \varsigma_{1}^{2}\right)
\end{array}\right.
$$

\section{A. Minimum BER Detector}

Since no CSI is available, we will design the data detector utilizing the difference ${ }^{1}$

$$
\Phi_{k}=\Gamma_{k}-\Gamma_{k-1} .
$$

\footnotetext{
${ }^{1}$ Different from the conventional different detector where the division between the two consecutive symbols is used, we here resort to the difference between the two consecutive symbols due to the inherent structure of ambient backscatter system.
}

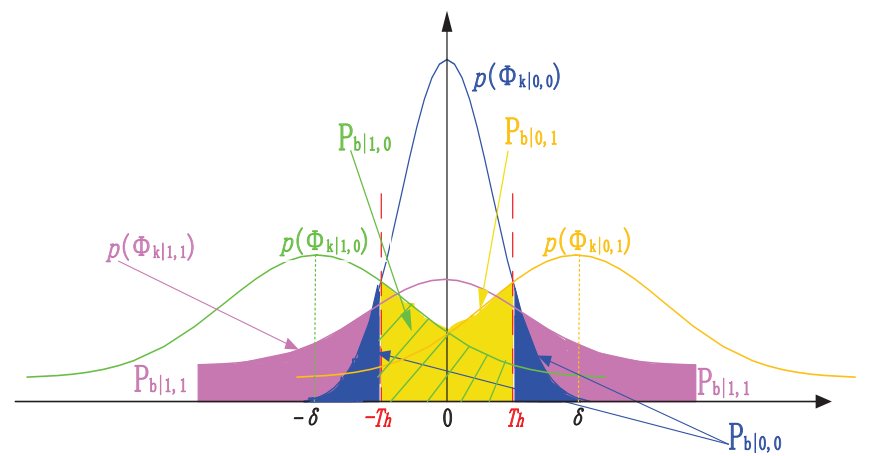

Fig. 4. PDFs of conditional random variables $\Phi_{k \mid 0,0}, \Phi_{k \mid 1,1}, \Phi_{k \mid 0,1}$ and $\Phi_{k \mid 1,0}$ and BER regions in the case of small and positive $\delta$.

Our goal is to design an optimal detector that can minimize the error probability, or equivalently, maximize the correct probability

$$
\begin{aligned}
\hat{A}(k) & =\arg \max _{A(k)=0,1} \operatorname{Pr}\left(\text { correct decision } \mid \Phi_{k}\right) \\
& =\arg \max _{A(k)=0,1} \operatorname{Pr}\left(A(k) \mid \Phi_{k}\right) .
\end{aligned}
$$

Since the transmit messages $A(k)=0$ and $A(k)=1$ are equiprobable, we can further simplify (19) as

$$
\hat{A}(k)=\arg \max _{A(k)=0,1} p\left(\Phi_{k} \mid A(k)\right) .
$$

The receiver given by (19) and (20) are well-known as maximum a posteriori probability (MAP) receiver and maximumlikelihood (ML) receiver respectively, which agrees with each other in the case of equiprobable transmit messages.

Since differential encoder is utilized by the tag, the message $A(k)=0$ (or $A(k)=1$ ) can be fully determined by $B(k)$ and $B(k-1)$. We can rewrite (20) as

$$
\hat{A}(k)=\arg \max _{A(k)=0,1} p\left(\Phi_{k} \mid B(k-1), B(k)\right) .
$$

For the cases of $B(k-1)=B(k)$ that indicates $A(k)=0$, we can further compute $\Phi_{k}$ as

$$
\Phi_{k}= \begin{cases}\Phi_{k \mid 0,0}=L_{k, 0}-L_{k-1,0}, & B(k-1)=B(k)=0 \\ \Phi_{k \mid 1,1}=L_{k, 1}-L_{k-1,1}, & B(k-1)=B(k)=1\end{cases}
$$

It can be readily checked that $\Phi_{k \mid 0,0} \sim \mathcal{N}\left(0,2 \varsigma_{0}^{2}\right)$ and $\Phi_{k \mid 1,1} \sim \mathcal{N}\left(0,2 \varsigma_{1}^{2}\right)$.

For the cases of $B(k-1) \neq B(k)$ that indicates $A(k)=1$, we can compute $\Phi_{k}$ as

$\Phi_{k}=$

$\begin{cases}\Phi_{k \mid 0,1}=\delta+L_{k, 1}-L_{k-1,0}, & B(k-1)=0, B(k)=1 \\ \Phi_{k \mid 1,0}=-\delta+L_{k, 0}-L_{k-1,1}, & B(k-1)=1, B(k)=0\end{cases}$

where

$$
\delta=\left(|\mu|^{2}-|h|^{2}\right) P_{s} .
$$

Clearly, $\Phi_{k \mid 0,1} \sim \mathcal{N}\left(\delta, \varsigma_{0}^{2}+\varsigma_{1}^{2}\right)$ and $\Phi_{k \mid 1,0} \sim \mathcal{N}\left(-\delta, \varsigma_{0}^{2}+\varsigma_{1}^{2}\right)$.

Let $p\left(\Phi_{k \mid 0,0}\right), p\left(\Phi_{k \mid 1,1}\right), p\left(\Phi_{k \mid 0,1}\right)$ and $p\left(\Phi_{k \mid 1,0}\right)$ denote the probability density functions (PDFs) of the conditional random variables $\Phi_{k \mid 0,0}, \Phi_{k \mid 1,1}, \Phi_{k \mid 0,1}$ and $\Phi_{k \mid 1,0}$, whose explicit expressions follow Gaussian distribution and are omitted for 


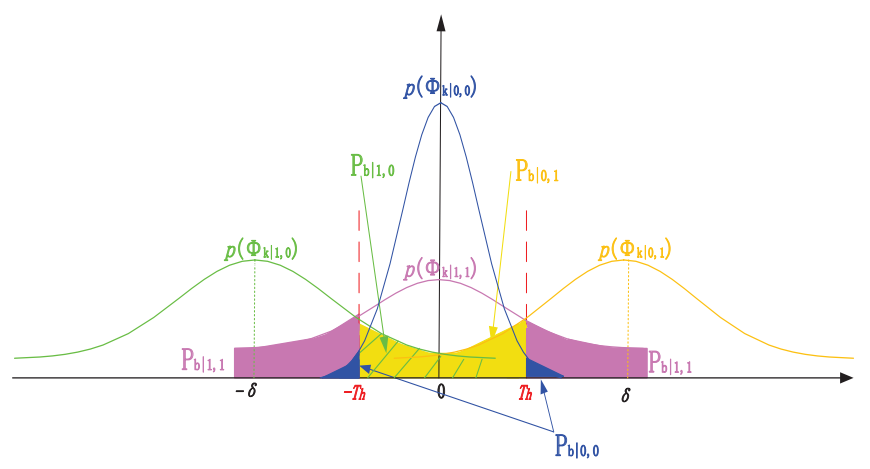

Fig. 5. PDFs of $p\left(\Phi_{k \mid 0,0}\right), p\left(\Phi_{k \mid 1,1}\right), p\left(\Phi_{k \mid 0,1}\right)$ and $p\left(\Phi_{k \mid 1,0}\right)$ and BER regions in the case of large and positive $\delta$.

brevity. Our ML detector (21) will choose one that can maximize the conditional PDF from the four candidate PDFs.

Fig. 4 shows the PDFs of the conditional random variables $\Phi_{k \mid 0,0}, \Phi_{k \mid 1,1}, \Phi_{k \mid 0,1}$ and $\Phi_{k \mid 1,0}$ in case of small and positive $\delta$. The corresponding BER regions of $P_{b \mid 0,0}, P_{b \mid 1,1}, P_{b \mid 0,1}$ and $P_{b \mid 1,0}$ are given in blue, purple, yellow and green shadowed part separately. Fig. 5 show the same PDFs and BER regions in the case of large and positive $\delta$.

\section{B. ML Decision Regions}

As shown in Fig. 4, if $\Phi_{k}$ falls into the region around zero, then we know $p\left(\Phi_{k \mid 0,0}\right)>p\left(\Phi_{k \mid 1,0}\right)$ or $p\left(\Phi_{k \mid 0,0}\right)>p\left(\Phi_{k \mid 0,1}\right)$, which indicates the optimal decision will be $\hat{A}(k)=0$; If $\Phi_{k}$ falls into the region around $\delta$ or larger than $\delta$, then $p\left(\Phi_{k \mid 1,1}\right)<$ $p\left(\Phi_{k \mid 1,0}\right)$ or $p\left(\Phi_{k \mid 1,1}\right)<p\left(\Phi_{k \mid 0,1}\right)$ holds, which indicates the optimal decision will be $\hat{A}(k)=1$.

The optimal threshold $T_{h}^{o p t}$ for the ML detector should satisfy

$$
p_{0}(x)=\left.p_{1}(x)\right|_{x=T_{h}^{o p t}},
$$

where

$$
\begin{aligned}
p_{0}(x) & =p\left(\Phi_{k \mid 0,0}\right)+p\left(\Phi_{k \mid 1,1}\right) \\
& =\frac{1}{\sqrt{4 \pi \varsigma_{0}^{2}}} e^{-\frac{x^{2}}{4 \varsigma_{0}^{2}}}+\frac{1}{\sqrt{4 \pi \varsigma_{1}^{2}}} e^{-\frac{x^{2}}{4 \varsigma_{1}^{2}}}, \\
p_{1}(x) & =p\left(\Phi_{k \mid 0,1}\right)+p\left(\Phi_{k \mid 1,0}\right) \\
& =\frac{1}{\sqrt{2 \pi \varsigma_{+}^{2}}}\left(e^{-\frac{(x-\delta)^{2}}{2 \varsigma_{+}^{2}}}+e^{-\frac{(x+\delta)^{2}}{2 \varsigma_{+}^{2}}}\right),
\end{aligned}
$$

and $\varsigma_{+}^{2}=\varsigma_{0}^{2}+\varsigma_{1}^{2}$.

Lemma 1: There exists at most one solution for (25) in the region $x \in(0,|\delta|)$.

Proof: Noting that

$$
\begin{aligned}
& \left(\varsigma_{0}^{2}+\varsigma_{1}^{2}+2 \sqrt{\varsigma_{0}^{2} \varsigma_{1}^{2}}\right)\left(\varsigma_{0}^{2}+\varsigma_{1}^{2}\right) \\
\geq & \left(2 \sqrt{\varsigma_{0}^{2} \varsigma_{1}^{2}}+2 \sqrt{\varsigma_{0}^{2} \varsigma_{1}^{2}}\right) \times 2 \sqrt{\varsigma_{0}^{2} \varsigma_{1}^{2}}=8 \varsigma_{0}^{2} \varsigma_{1}^{2},
\end{aligned}
$$

where the equality can be achieved when and only when $\varsigma_{0}^{2}=$ $\varsigma_{1}^{2}$. Therefore, we can find

$$
\frac{\left(\sqrt{\varsigma_{0}^{2}}+\sqrt{\varsigma_{1}^{2}}\right)^{2}}{2 \varsigma_{0}^{2} \varsigma_{1}^{2}} \geq \frac{4}{\varsigma_{0}^{2}+\varsigma_{1}^{2}},
$$

which indicates

$$
\left(\frac{1}{\sqrt{2 \varsigma_{0}^{2}}}+\frac{1}{\sqrt{2 \varsigma_{1}^{2}}}\right)^{2} \geq\left(\frac{2}{\sqrt{\varsigma_{0}^{2}+\varsigma_{1}^{2}}}\right)^{2} .
$$

We can thus obtain

$$
\begin{aligned}
\left.p_{0}(x)\right|_{x=0} & =\frac{1}{\sqrt{4 \pi \varsigma_{0}^{2}}}+\frac{1}{\sqrt{4 \pi \varsigma_{1}^{2}}} \\
& \geq \frac{2}{\sqrt{2 \pi\left(\varsigma_{0}^{2}+\varsigma_{1}^{2}\right)}} \geq\left. p_{1}(x)\right|_{x=0} .
\end{aligned}
$$

On the other hand, it can be readily checked that $p_{1}(x)$ is a monotone increasing function in the region $(0,|\delta|)$. Noticing that $p_{0}(x)$ is strictly monotone decreasing function when $x \in$ $(0,|\delta|)$ and utilizing (31), we can claim that if there exists an intersection point in the region $(0,|\delta|)$ for the function curves $p_{0}(x)$ and $p_{1}(x)$, it should be only one.

Therefore, our ML decision rule is: decode $\hat{A}(k)=0$ when $0 \leq\left|\Phi_{k}\right|<T_{h}$, and $\hat{A}(k)=1$ otherwise. Here, $T_{h}$ represents a properly chosen threshold.

Unfortunately, there does not exist any closed-form solution for equation (25). Therefore, we resort to approximate approaches and have the following theorem.

Theorem 1: The solution for equation (25) can be approximated by

$$
T_{h}^{a p x}=\frac{|\delta|}{2}+\frac{\varsigma_{+}^{2}}{|\delta|} \ln \left(1+\sqrt{1-e^{-\delta^{2} / \varsigma_{+}^{2}}}\right) .
$$

Proof: The function $p_{0}(x)$ of the two PDFs' sum can be approximated by

$$
\tilde{p}_{0}(x)=\frac{2}{\sqrt{2 \pi \varsigma_{+}^{2}}} e^{-\frac{x^{2}}{2 \varsigma_{+}^{2}}} .
$$

We can then rewrite (25) as

$$
2 e^{-\frac{x^{2}}{2 \varsigma_{+}^{2}}}=e^{-\frac{(x-\delta)^{2}}{2 \varsigma_{+}^{2}}}+e^{-\frac{(x+\delta)^{2}}{2 \varsigma_{+}^{2}}},
$$

which can be further simplified as

$$
e^{\frac{x \delta}{\varsigma_{+}^{2}}}+e^{-\frac{x \delta}{\varsigma_{+}^{2}}}=2 e^{\frac{\delta^{2}}{2 \varsigma_{+}^{2}}} .
$$

Treating $e^{\frac{x \delta}{\varsigma_{+}^{2}}}$ as a variable, we can obtain from (35)

$$
e^{\frac{\delta^{2}}{2 \varsigma_{+}^{2}}}+\sqrt{e^{\frac{\delta 2}{\varsigma_{+}^{2}}}-1}=\left\{\begin{array}{cc}
e^{\frac{x \delta}{\varsigma_{+}^{2}}}, & \delta>0 \\
e^{-\frac{x \delta}{\varsigma_{+}^{2}}}, & \delta<0
\end{array}\right.
$$

Taking logarithm operations for both sides of (36) can produce

$$
x=\left\{\begin{array}{cc}
\frac{\delta}{2}+\frac{\varsigma_{+}^{2}}{\delta} \ln \left(1+\sqrt{1-e^{-\delta^{2} / \varsigma_{+}^{2}}}\right), & \delta>0 \\
-\frac{\delta}{2}-\frac{\varsigma_{+}^{2}}{\delta} \ln \left(1+\sqrt{1-e^{-\delta^{2} / \varsigma_{+}^{2}}}\right), & \delta<0
\end{array}\right.
$$

which can result in (32). 
Noticing that at high SNR, the first item in (32) is much larger than the second one, we can have the following conclusion.

Corollary 1: At high SNR, the optimal threshold $T_{h}^{o p t}$ can be approximated by $|\delta| / 2$.

Remark 2: According to Corollary 1, the practical threshold can be set as $E\left(\left|\Phi_{k}\right|\right)$ as an alternative to $T_{h}^{o p t}$ in high SNR because $E\left(\left|\Phi_{k}\right|\right) \approx|\delta| / 2$ when $K$ is large.

\section{Performance Analysis}

\section{A. BER Performance}

Noting that $B(k-1)=0$ or $B(k-1)=1$ are equiprobable and so are $B(k)=0$ or $B(k)=1$. Hence, the BER can be found as

$$
P_{b}=\frac{1}{4}\left(P_{b \mid 0,0}+P_{b \mid 1,1}+P_{b \mid 0,1}+P_{b \mid 1,0}\right),
$$

where

$$
\begin{aligned}
P_{b \mid 0,0} & =\operatorname{Pr}(\hat{A}(k)=1 \mid B(k-1)=0, B(k)=0) \\
& =1-\int_{-T_{h}}^{T_{h}} p\left(\Phi_{k \mid 0,0}\right) \mathrm{d} \Phi_{k \mid 0,0}=2 Q\left(\frac{T_{h}}{\sqrt{2 \varsigma_{0}^{2}}}\right), \\
P_{b \mid 1,1} & =\operatorname{Pr}(\hat{A}(k)=1 \mid B(k-1)=1, B(k)=1), \\
& =1-\int_{-T_{h}}^{T_{h}} p\left(\Phi_{k \mid 1,1}\right) \mathrm{d} \Phi_{k \mid 1,1},=2 Q\left(\frac{T_{h}}{\sqrt{2 \varsigma_{1}^{2}}}\right), \\
P_{b \mid 0,1} & =\operatorname{Pr}(\hat{A}(k)=0 \mid B(k-1)=0, B(k)=1) \\
& =Q\left(\frac{-T_{h}-\delta}{\sqrt{\varsigma_{0}^{2}+\varsigma_{1}^{2}}}\right)-Q\left(\frac{T_{h}-\delta}{\sqrt{\varsigma_{0}^{2}+\varsigma_{1}^{2}}}\right), \\
P_{b \mid 1,0}= & \operatorname{Pr}(\hat{A}(k)=0 \mid B(k-1)=1, B(k)=0) \\
= & Q\left(\frac{-T_{h}+\delta}{\sqrt{\varsigma_{0}^{2}+\varsigma_{1}^{2}}}\right)-Q\left(\frac{T_{h}+\delta}{\sqrt{\varsigma_{0}^{2}+\varsigma_{1}^{2}}}\right) .
\end{aligned}
$$

Here, the $Q$ function is defined as

$$
Q(x)=\frac{1}{\sqrt{2 \pi}} \int_{x}^{\infty} e^{-\frac{t^{2}}{2}} \mathrm{~d} t .
$$

Due to symmetry, we can have $P_{b \mid 0,1}=P_{b \mid 1,0}$. Therefore, substituting (39), (40), (41) and (42) into (38), we can obtain the BER expression as

$$
\begin{aligned}
P_{b}=\frac{1}{2} Q & \left(\frac{T_{h}}{\sqrt{2 \varsigma_{0}^{2}}}\right)+\frac{1}{2} Q\left(\frac{T_{h}}{\sqrt{2 \varsigma_{1}^{2}}}\right) \\
& +\frac{1}{2} Q\left(\frac{-T_{h}+\delta}{\sqrt{\varsigma_{0}^{2}+\varsigma_{1}^{2}}}\right)-\frac{1}{2} Q\left(\frac{T_{h}+\delta}{\sqrt{\varsigma_{0}^{2}+\varsigma_{1}^{2}}}\right) .
\end{aligned}
$$

\section{B. Upper and Lower Bounds for BER}

Next, we assume high SNR and choose $T_{h}=|\delta| / 2$. Thus, it can be found that

$$
\begin{aligned}
& P_{b \mid 0,0}=2 Q\left(\frac{|\delta| / 2}{\sqrt{2 \varsigma_{0}^{2}}}\right) \approx 2 Q\left(\frac{\Delta_{\mu h}}{4|h|} \sqrt{\gamma N}\right), \\
& P_{b \mid 1,1}=2 Q\left(\frac{|\delta| / 2}{\sqrt{2 \varsigma_{1}^{2}}}\right) \approx 2 Q\left(\frac{\Delta_{\mu h}}{4|\mu|} \sqrt{\gamma N}\right),
\end{aligned}
$$

and

$$
\begin{aligned}
P_{b \mid 0,1} & =Q\left(\frac{|\delta| / 2}{\sqrt{\varsigma_{0}^{2}+\varsigma_{1}^{2}}}\right)-Q\left(\frac{3|\delta| / 2}{\sqrt{\varsigma_{0}^{2}+\varsigma_{1}^{2}}}\right)=P_{b \mid 1,0} \\
& \approx Q\left(\frac{\Delta_{\mu h}}{2 \sqrt{2} \sqrt{\Xi_{\mu h}}} \sqrt{\gamma N}\right)-Q\left(\frac{3 \Delta_{\mu h}}{2 \sqrt{2} \sqrt{\Xi_{\mu h}}} \sqrt{\gamma N}\right),
\end{aligned}
$$

where $\gamma=P_{s} / N_{w b}$ and

$$
\Delta_{\mu h}=\left.|| \mu\right|^{2}-\left.|h|^{2}\left|, \quad \Xi_{\mu h}=\right| h\right|^{2}+|\mu|^{2} .
$$

Utilizing the simple but good approximation for $Q(x)$ [11]

$$
Q(x) \approx \frac{1}{12} e^{-\frac{x^{2}}{2}}+\frac{1}{4} e^{-\frac{2 x^{2}}{3}}, \quad x>0 .
$$

and substituting (45), (46), and (47) into (38) will produce

$$
\begin{aligned}
& \left.P_{b}\right|_{T_{h}=|\delta| / 2} \triangleq P_{b, L} \approx \\
& \frac{1}{24} e^{-\frac{\Delta_{\mu h}^{2} \gamma N}{32|h|^{2}}}+\frac{1}{8} e^{-\frac{\Delta_{\mu h}^{2} \gamma N}{24|h|^{2}}}+\frac{1}{24} e^{-\frac{\Delta_{\mu h}^{2} \gamma N}{32|\mu|^{2}}}+\frac{1}{8} e^{-\frac{\Delta_{\mu h}^{2} \gamma N}{24|\mu|^{2}}} \\
& +\frac{1}{24} e^{-\frac{\Delta_{\mu h}^{2} \gamma N}{16 \Xi_{\mu h}}}+\frac{1}{8} e^{-\frac{\Delta_{\mu h}^{2} \gamma N}{12 \Xi_{\mu h}}}-\frac{1}{24} e^{-\frac{9 \Delta_{\mu h}^{2} \gamma N}{16 \Xi_{\mu h}}}-\frac{1}{8} e^{-\frac{3 \Delta_{\mu h}^{2} \gamma N}{4 \Xi_{\mu h}}},
\end{aligned}
$$

where $\triangleq$ denotes definition.

Define

$$
\Xi_{l}=\min \left(|h|^{2},|\mu|^{2}\right), \quad \Xi_{u}=\max \left(|h|^{2},|\mu|^{2}\right) .
$$

It can be readily checked that

$$
2 \Xi_{l} \leq \Xi_{\mu h} \leq 2 \Xi_{u}
$$

Since the function $e^{-\frac{1}{x}}$ is an increasing function with $x$, we can find

$$
\begin{aligned}
& P_{b, L} \leq \frac{1}{8} e^{-\frac{\Delta_{\mu h}^{2} \gamma N}{32 \Xi_{u}}}+\frac{3}{8} e^{-\frac{\Delta_{\mu h}^{2} \gamma N}{24 \Xi_{u}}} \triangleq P_{u b}, \\
& P_{b, L} \geq \frac{5}{48} e^{-\frac{\Delta_{\mu h}^{2} \gamma N}{32 \Xi_{l}}}+\frac{5}{16} e^{-\frac{\Delta_{\mu h}^{2} \gamma N}{24 \Xi_{l}}} \triangleq P_{l b},
\end{aligned}
$$

where $P_{u b}$ and $P_{l b}$ defined as the corresponding items represent the upper and lower bounds for the BER $P_{b}$ when the threshold is chosen as $T_{h}=\delta \mid / 2$, respectively.

Remark 3: It can be observed from (50), (53) and (54) that the uplink BER for ambient backscatter communication systems is determined by the four factors: $\operatorname{SNR} \gamma$, the number for averaging $N$, the channel difference $\Delta_{\mu h}$, and the channels $|h|$ and $|\mu|$. In addition, the BER can be considered as an decreasing function of variables $\gamma, N$ and $\Delta_{\mu h}$, and as a increasing function of variables $|h|$ and $|\mu|$.

\section{Simulation Results}

In this section, we numerically examine the proposed studies. The noise variance $N_{w b}=1$ is set as 1 and $K$ is chosen as 100 .

Fig. 6 gives the BER versus SNR for two detection thresholds. We first set the number of averaging samples $N$ as 20 and increase the transmit SNR from 0dB to 30dB. For each SNR, we obtain the optimal threshold $T_{h}^{\text {opt }}$ from (25) for our detector and find its average BER after $10^{4}$ Monte Carlo simulations. We then set the threshold as $E\left(\left|\Phi_{k}\right|\right)$ for the detector, and get the simulated corresponding BER. We also set the threshold 


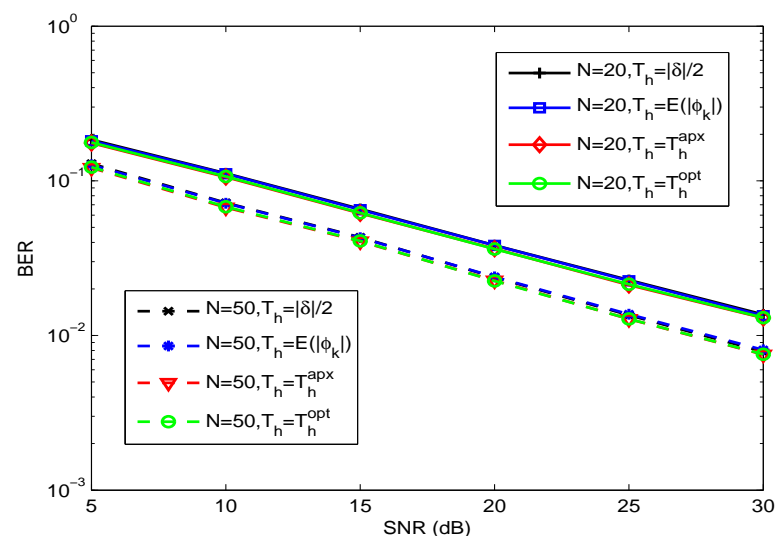

Fig. 6. BER versus transmit SNR.

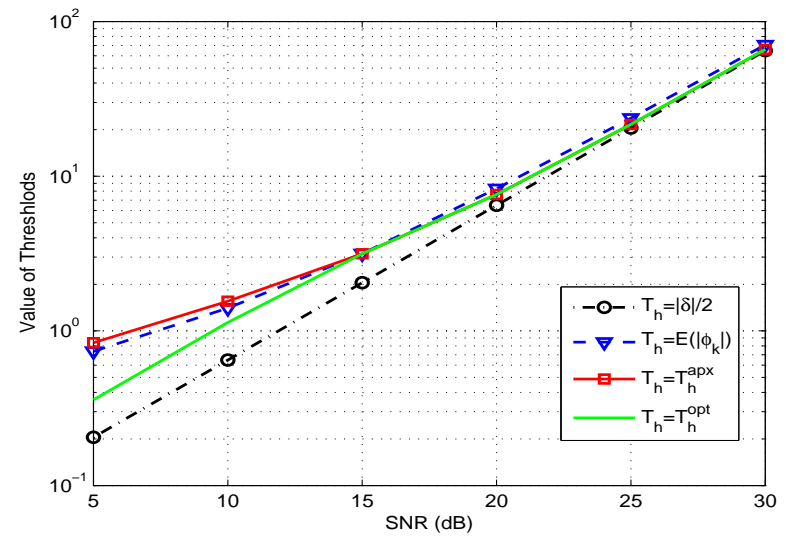

Fig. 7. Four thresholds $T_{h}^{o p t}, T_{h}^{a p x},|\delta| / 2$ and $E\left(\left|\Phi_{k}\right|\right)$ versus SNR.

as $T_{h}^{a p x}$ and $|\delta| / 2$ respectively, and find the corresponding BERs. We then set $N=50$ and repeat the above process. It can be seen from Fig. 6 that a larger $N$ can lead to reduced BER. It can also be found that the optimal threshold $T_{h}^{o p t}$ slightly outperforms the other three thresholds $E\left(\left|\Phi_{k}\right|\right), T_{h}^{a p x}$ and $|\delta| / 2$.

Fig. 7 depicts the curves of four different thresholds $T_{h}^{o p t}$, $T_{h}^{a p x},|\delta| / 2$ and $E\left(\left|\Phi_{k}\right|\right)$ versus SNR. At low SNR, we can find that there exists difference between the curves of threshold $T_{h}^{o p t}$ and that of $|\delta| / 2$. However, when SNR is greater than $20 \mathrm{~dB}$, the difference vanishes, which agrees with our Theorem 1. Besides, the value $E\left(\left|\Phi_{k}\right|\right)$ (or $T_{h}^{a p x}$ ) can be a good approximate for $T_{h}^{o p t}$ at high SNR, while there exists a large gap between $T_{h}^{o p t}$ and $E\left(\left|\Phi_{k}\right|\right)$ (or $T_{h}^{a p x}$ ) at low SNR.

Fig. 8 shows the curves of BER versus $\mathrm{N}$ when $\mathrm{SNR}=30 \mathrm{~dB}$. The detectors choose the optimal threshold $T_{h}^{o p t}$ and the corresponding BER are plotted. For comparison, the upper bound (54) and lower bound (53) of BER, as well as the approximate BER (50), are also plotted. Clearly, our approximate theoretical BER approaches simulated BER very well, and the upper bound and the lower bound of BER is close to the simulated BER. It can also be seen that the values of $N$ can lower the BER effectively when $N$ is small, and there exist error floors in the BER curves when $N$ is greater than 120.

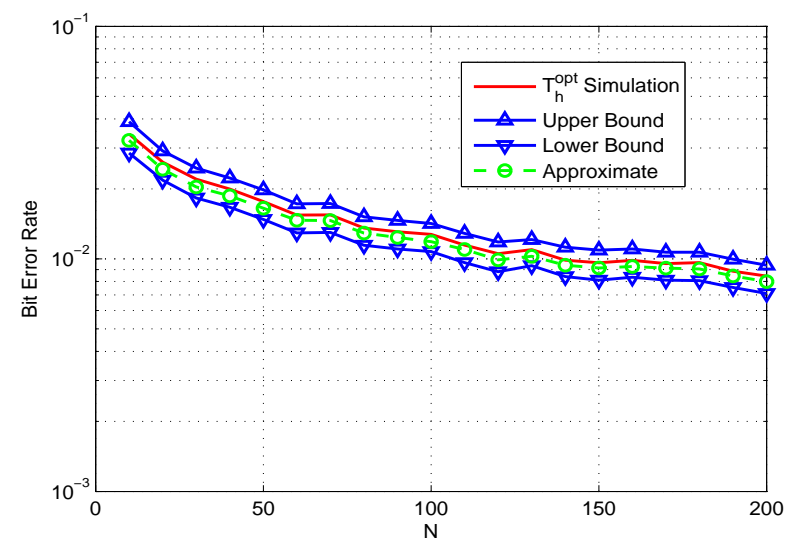

Fig. 8. BER versus $N$, the number of samples for averaging.

\section{CONCLUSION}

Ambient backscatter is a new wireless communication technology with good research potential and huge market value as well as many open problems. In this paper, we built up the theoretical model for the communication system with ambient backscatter and differential encoder. Furthermore, the detector that can minimize the BER was suggested and the approximate optimal detection threshold was derived. The BER performance versus SNR was also obtained and both upper and lower BER bounds were derived in an uncomplicated and closed form. Finally, simulation results were provided to corroborate our proposed studies.

\section{REFERENCES}

[1] L. Xie, Y. Yin, A. V. Vasilakos, and S. Lu, "Managing RFID data: challenges, opportunities and solutions," IEEE Commun. Surveys and Tutorials, vol. 16, no. 3, pp. 1294-1311, Aug. 2014.

[2] D. M. Dobkin, The RF in RFID: Passive UHF RFID in Practice. Newnes (Elsevier), 2008.

[3] H. Stockman, "Communication by means of reflected power," Proc. IRE, pp. 1196-1204, Oct. 1948.

[4] C. Boyer and S. Roy, "Backscatter communication and RFID: coding, energy and MIMO analysis," IEEE Trans. Commun., vol. 62, no.3, pp. 770-785, Mar. 2014.

[5] W. Saad, X. Zhou, Z. Han, and H. V. Poor, "On the physical layer security of backscatter wireless systems," IEEE Trans. Wireless Commun. vol. 13, no. 2 pp. 3442-3451, Dec. 2014.

[6] J. Kimionis, A. Bletsas, and J. N. Sahalos, "Increased range bistatic scatter radio," IEEE Trans. Commun., vol. 62, no. 3, pp. 1091-1104, Mar. 2014.

[7] V. Liu, A. Parks, V. Talla, S. Gollakota, D. Wetherall, and J. R. Smith, "Ambient backscatter: wireless communication out of thin air," in Proc. ACM SIGCOMM, Hong Kong, China, 2013, pp. 1-13.

[8] B. Kellogg, A. Parks, S. Gollakota, J. R. Smith, and D. Wetherall, "Wi-Fi Backscatter: Internet connectivity for RF-powered devices," in Proc. ACM SIGCOMM, Chicago, USA, 2014, pp. 1-12.

[9] S. Gollakota, M. S. Reynolds, J. R. Smith, and D. Wetherall, "The emergence of RF-powered computing," IEEE Computer, vol. 47, pp. 32-39, Jan. 2014.

[10] F. Fuschini, C. Piersanti, F. Paolazzi, and G. Falciasecca, "Analytical approach to the backscattering from UHF RFID transponder," IEEE Antennas Propag. Lett., vol. 7, pp. 33-35, 2008.

[11] M. Chiani, D. Dardari, and M. K. Simon, "New exponential bounds and approximations for the computation of error probability in fading channels," IEEE Trans. Wireless Commun., vol. 2, pp. 840-845, July 2003. 